\title{
Holographic Calculation of the Magneto-Transport Coefficients in the Dirac Semimetals
}

\begin{abstract}
M. RogatKo* AND K.I. WYSOKIŃSKI
Institute of Physics, M. Curie-Skłodowska University, pl. M. Curie-Skłodowskiej 1, 20-031 Lublin, Poland

We use the holographic analogy to calculate magneto-thermoelectric transport coefficients of the strongly interacting electrons. The examples of the real materials which supposedly contain strongly interacting electron-hole fluid include graphene, the topological insulators or three-dimensional compounds with the Dirac spectrum. In such systems the particles (electron and holes if the Fermi energy is located close to the Dirac point) behave as a strongly interacting fluid. This has been observed in graphene, where experimental signatures of the strong interactions have been observed, and is assumed to be valid for other systems. On the gravity side we have modeled the transport of such materials by two interacting vector fields. According to the gauge-gravity interpretation two vector fields represent electron and hole currents flowing in the system under the effect of electric field. The axionic field introduced into the gravity action provides momentum relaxation and gives finite values of DC transport coefficients. We have calculated charge conductivity and thermal conductivity tensors and tensor of the Wiedemann-Franz ratio. Our results favorably compare with the existing experimental data.
\end{abstract}

DOI: 10.12693/APhysPolA.135.51

PACS/topics: classical general relativity; canonical formalism, Lagrangians, and variational principles; galvanomagnetic and other magnetotransport effects

\section{Introduction}

There exist various theoretical methods of calculation of the (charge and energy) currents which appear in the system in response to the applied forces: whether electric field $\boldsymbol{E}$ or temperature gradient $\nabla T$. Typically we are interested in small disturbances $(\boldsymbol{E}$ and $\nabla T)$ so it suffices to calculate the currents to the linear order in the forces. The corresponding coefficients relating the currents to the forces are known as kinetic coefficients. Their knowledge is enough to get the corresponding transport coefficients. Among many standard methods used to calculate them one can mention: the Drude approach, the Boltzmann equation and various Kubo-Greenwood linear response techniques based on the Green functions.

Recently still another approach has appeared in the literature. It is related to the so called holographic duality [1], also known [2] as gauge-gravity duality or anti-de Sitter/condensed matter theory correspondence (AdS/CMT correspondence). This method is based on the important development on the gravity side [1] which shows that one can calculate properties of the real systems using the Einstein theory of gravity but formulated not in our real world space-time, but in the mentioned AdS space-time which is characterized by the existence of the boundary in spatial direction $r$. The condensed matter system is supposed to leave on the boundary of the gravity space-time [3].

There exist a number of recent papers [4-8] in which the transport properties of the graphene have been studied by means of holographic duality. The important reservation is that the system studied by means of

*corresponding author; e-mail: rogat@kft.umcs.lublin.pl holography has to be strongly interacting one. This is related to the so-called holographic renormalization, which means that the lowest order calculations on the gravity side correspond to the strong coupling results on the non-gravity field theory side [9]. As far as graphene is concerned there have been found experimental signatures [10] pointing out that the system really falls into the strong coupling limit if it is tuned so that the Fermi energy is at or close to the Dirac point. In this paper we assume that the same is true for other two-dimensional systems with the Dirac spectrum and also for threedimensional counterparts of the graphene known as the Dirac semimetals (DSM).

In recent paper [11] we have used the holographic approach to analyze the properties of the Dirac semimetals. Here we provide additional results and extended discussion of the possible generalization of the WiedemannFranz ratio to study the strongly interacting electrons in a magnetic field.

\section{The model and approach}

To learn about the transport in condensed matter systems with the Dirac-like spectrum by means of gravity theory one has to know that the particles are really very strongly interacting, so that one rather speaks about the liquid and not individual electrons or holes. As mentioned, the recent experiments [10] and other studies on graphene [12] show that indeed this is the case if the system is tuned close to the Dirac point, which is the particle-hole symmetry point. Here we assume that all known systems with the linear spectrum close to the Fermi energy are strongly interacting. That this assumption might be true one can judge from the very low zero temperature resistance of topological semimetal ZrSiS [13, 14] and other systems [15] with linear spectrum. 
To start we take the gravitational action in $(4+1)$-dimensions

$$
\begin{aligned}
S & =\int \sqrt{-g} \mathrm{~d}^{5} x\left(R+\frac{6}{L^{2}}-\frac{1}{2} \nabla_{\mu} \phi_{i} \nabla^{\mu} \phi^{i}\right. \\
& \left.-\frac{1}{4} F_{\mu \nu} F^{\mu \nu}-\frac{1}{4} B_{\mu \nu} B^{\mu \nu}-\frac{\alpha}{4} F_{\mu \nu} B^{\mu \nu}\right),
\end{aligned}
$$

where $\left.F_{\mu \nu}=2 \nabla_{[\mu} A_{\nu}\right]$ stands for the ordinary Maxwell field strength tensor, while the second $U(1)$-gauge field $B_{\mu \nu}$ is given by $B_{\mu \nu}=2 \nabla_{\left[\mu B_{\nu}\right]} . \alpha$ is a coupling constant between two gauge fields. In turn for the gauge fields we assume the components $A_{\mu}(r) \mathrm{d} x^{\mu}=a(r) \mathrm{d} t+$ $\frac{B}{2}(x \mathrm{~d} y-y \mathrm{~d} x)$ and $B_{\mu}(r) \mathrm{d} x^{\mu}=b(r) \mathrm{d} t+\frac{B_{\text {add }}}{2}(x \mathrm{~d} y-y \mathrm{~d} x)$, where by the symbol $B$ we have denoted a background magnetic field and $B_{a d d}$ is the magnetic field of the additional $U(1)$-gauge field coupled to the Maxwell one. At the end of calculations one assumes that $B_{\text {add }}=B$. The charge current densities in the theory are given by $J_{(F)}^{\mu}=\sqrt{-g}\left(F^{\mu r}+\alpha / 2 B^{\mu r}\right)$ and $J_{(B)}^{\mu}=\sqrt{-g}\left(B^{\mu r}+\right.$ $\left.\alpha / 2 F^{\mu r}\right)$, where the right-hand sides are evaluated at the space-time boundary, when $r \rightarrow \infty$. The heat current density is calculated from the identity derived in $[6,11]$ involving the time-directed Killing vector and a two form $\tilde{G}_{\nu \rho}$ leading to the following expression for the heat current: $Q^{i}=2 \sqrt{-g} \tilde{G}_{i r}$.

To calculate conductivities in the holographic approach one has to disturb the system. On the gravity side this is done by modifying both the field components $a(r)$ and $b(r)$ (corresponding to scalar potentials of the nonrelativistic theory) and one has to allow for modifications of the gravitational background, i.e. the metric. The latter is modified in time $(t)$ and bulk gravity $(r)$ directions. The charge and heat currents are expressed in terms of the assumed fields and the metric tensor perturbation $\delta g_{t j}^{(h)}$ as

$$
\begin{aligned}
& J_{(F)}^{i}(\infty)=r_{h}\left[-\frac{\tilde{Q}_{(F)}}{r_{h}^{3}} \delta^{i j} \delta g_{t j}^{(h)}\right. \\
& \left.+\frac{\delta g_{t j}^{(h)}}{2 r_{h}^{2}} \epsilon^{i j}\left(B+\frac{\alpha}{2} B_{\text {add }}\right)+\delta^{i j}\left(E_{j}+\frac{\alpha}{2} B_{j}\right)\right] \\
& J_{(B)}^{i}(\infty)=r_{h}\left[-\frac{\tilde{Q}_{(B)}}{r_{h}^{3}} \delta^{i j} \delta g_{t j}^{(h)}\right. \\
& \left.\quad+\frac{\delta g_{t j}^{(h)}}{2 r_{h}^{2}} \epsilon^{i j}\left(B_{\text {add }}+\frac{\alpha}{2} B\right)+\delta^{i j}\left(B_{j}+\frac{\alpha}{2} E_{j}\right)\right] \\
& \tilde{Q}^{i}(\infty)=-4 \pi T \delta^{i j} \delta g_{t j}^{(h)} .
\end{aligned}
$$

The resulting perturbation of the metric tensor is calculated from the Einstein equations and can be written as [11]:

$$
\begin{gathered}
\delta g_{t j}^{(h)}=-\frac{A}{A^{2}+C^{2}}\left(K_{i}+D_{i}+H_{i}\right) \delta^{i j} \\
+\frac{C}{A^{2}+C^{2}}\left(K_{i}+D_{i}+H_{i}\right) \epsilon^{i j},
\end{gathered}
$$

where $\epsilon^{i j}$ is the two-dimensional antisymmetric tensor with $\epsilon^{x y}=-1$. The symbols have the following meaning:

$$
\begin{aligned}
& A=\frac{\tilde{B}^{2}+12 \beta^{2} r_{h}^{2}}{2 r_{h}^{3}}, \\
& K_{m}=4\left(Q_{(F)}\left(r_{h}\right) E_{m}+Q_{(B)}\left(r_{h}\right) B_{m}\right) \\
& \quad+2 \alpha\left(Q_{(F)}\left(r_{h}\right) B_{m}+Q_{(B)}\left(r_{h}\right) E_{m}\right), \\
& C=\frac{B}{r_{h}^{2}}\left(2 Q_{(F)}\left(r_{h}\right)+\alpha Q_{(B)}\left(r_{h}\right)\right) \\
& \quad+\frac{B_{\text {add }}}{r_{h}^{2}}\left(2 Q_{(B)}\left(r_{h}\right)+\alpha Q_{(F)}\left(r_{h}\right)\right), \\
& D_{m}=16 \pi T \xi_{m} r_{h} 3, \\
& H_{m}=-2 B \epsilon_{m k} E^{k} r_{h}-2 B_{\text {add }} \epsilon_{m k} B^{k} r_{h} .
\end{aligned}
$$

For the brevity of the notation we set $\tilde{B}^{2}=B^{2}+B_{\text {add }}^{2}+$ $\alpha B B_{\text {add }}, \xi_{m}=(\nabla T)_{m} / T$ and $E_{m}, B_{m}$ are electric fields related to two sectors in the action.

The axionic field $\phi$ in the action plays a very important role on the gravity and field theory sides. On the gravity side it means that in order to analyze the solution in detail one needs special black hole solution [11]. The explicit analysis of the model requires dyonic black hole solutions. In the probe limit the fields do not change the gravity background and the following ansatz for the static five-dimensional topological black brane with planar symmetry is appropriate. It has the form

$$
\begin{gathered}
\mathrm{d} s^{2}=-f(r) \mathrm{d} t^{2}+\frac{\mathrm{d} r^{2}}{f(r)} \\
+r^{2}\left(\mathrm{~d} x^{2}+\mathrm{d} y^{2}+\mathrm{d} z^{2}\right),
\end{gathered}
$$

where $f(r)=\frac{r^{2}}{2 L^{2}}-\frac{m}{r^{2}}$, and $m$ is constant. The Hawking temperature is provided by the expression

$$
T=\left.\frac{1}{4 \pi} f^{\prime}(r)\right|_{r \rightarrow r_{h}}=\frac{1}{2 \pi} r_{h} .
$$

The radius $L$ of the AdS space-time is set equal to one.

On the field theory side the importance of the axionic field results from the fact that it provides a momentum dissipation $[4,5]$ in the system and thus allows for finite values of the direct current transport coefficients, which are discussed in the next section.

\section{Results}

With two external fields marked by $F$ and $B$ we end up with three currents $\boldsymbol{J}_{F}, \boldsymbol{J}_{B}, \boldsymbol{Q}$. They correspond to three vector fields $\boldsymbol{E}_{F}=\left(E_{F}^{x}, E_{F}^{y}\right), \boldsymbol{E}_{B}=$ $\left(E_{B}^{x}, E_{B}^{y}\right), \boldsymbol{\xi}$, where $\boldsymbol{E}_{F}$ and $\boldsymbol{E}_{B}$ are interpreted as electric fields in sectors $F$ and $B$ respectively, while $\boldsymbol{\xi}=$ $-\nabla T / T$ represents thermal force due to the temperature gradient. The two electric currents are discussed in the following as resulting from electrons and holes in the system. We thus define the matrix of kinetic coefficients 


$$
\left(\begin{array}{c}
J_{F}^{i} \\
J_{B}^{i} \\
Q^{i}
\end{array}\right)=\left(\begin{array}{ccc}
\sigma_{j_{F F}}^{i} & \sigma_{j_{F B}}^{i} & \alpha_{j_{F}}^{i} T \\
\sigma_{j_{B F}}^{i} & \sigma_{j_{B B}}^{i} & \alpha_{j_{B} T}^{i} T \\
\alpha_{j_{F}}^{i} T & \alpha_{j_{B}}^{i} T & \kappa_{0 j}^{i} T
\end{array}\right)\left(\begin{array}{c}
E_{F}^{j} \\
E_{B}^{j} \\
\xi^{j}
\end{array}\right)
$$

with $i, j=x, y$. Note that the magnetic field is directed along the $z$ axis, so we shall not be able to discuss the effects connected with $\boldsymbol{E} \cdot \boldsymbol{B}$ or $\boldsymbol{\xi} \cdot \boldsymbol{B}$ terms, sometimes called "axial-gravitational anomalies" [16].

We shall not write down the explicit formulae for the matrix elements $\sigma_{a b}^{i j}$. The analysis of the results shows that both the magneto-conductance and magnetoresistance are sensitive functions of the holographic dissipation parameter $\beta$, which on the condensed matter side we interpret as the inverse mobility $\mu$ of carriers. Precisely in the studied 3D system one can identify

$$
\mu^{2}=\frac{1}{12 \beta^{2} r_{h}^{2}} .
$$

This identification allows us to write the magnetoconductance in terms of $\mu B$ terms characteristic for the Drude-Boltzmann approach. The holographic analysis shows that the above mobility is inversely proportional to temperature if one identifies $T=r_{h} /(2 \pi)$.

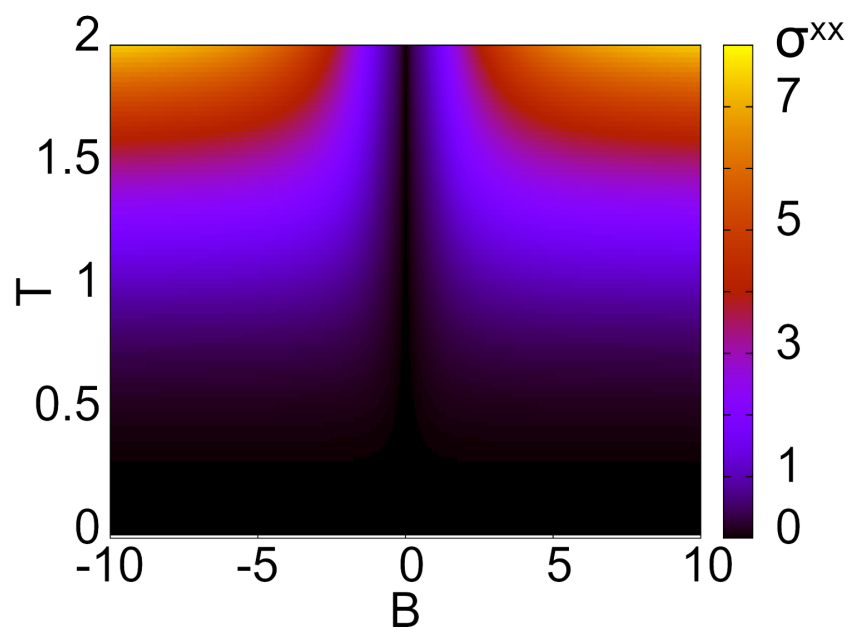

Fig. 1. The dependence of $\sigma^{x x}$ on the magnetic field $B$ and temperature $T$. We set $r_{h}=T$.

To calculate and analyze the charge carrier density dependence of the thermal conductivity of graphene with no external magnetic field the authors [8] have diagonalized the full matrix (which, in our notation, contained only elements $\left.\sigma_{F F(B B)}^{x x}\right)$ and got a good agreement with experiment. The analogous analysis is performed here for the model with the magnetic field. Figure 1 shows the dependence of $\sigma^{x x}$ on both temperature and magnetic field. Thermal conductivity $\kappa^{i j}$ tensor is defined as:

$$
Q_{i}=-\kappa^{i j} T \xi_{j},
$$

under the proviso that no currents flow $\left(J_{F}=0, J_{B}=0\right)$ in the system. The dependence of the diagonal part of it on the magnetic field for a few values of parameter $r_{h} \propto T$ is shown in Fig. 2b.
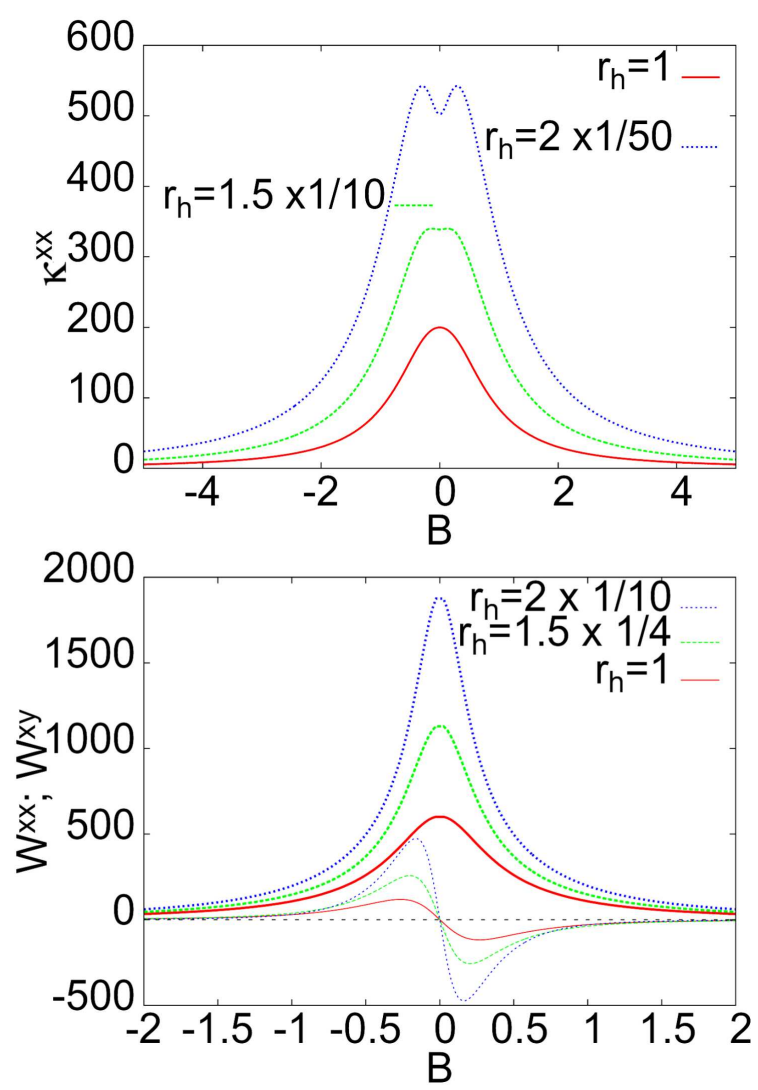

Fig. 2. (upper part) Magnetic field dependence of the thermal conductivity coefficient $\kappa^{x x}$ close to the charge neutrality point $\left(Q_{F}=Q_{B}=0.1\right)$ for three values of the temperature $T=1,1.5,2$. For better visibility the values of $\kappa^{x x}$ have been divided by 50 and 10 for $r_{h}=2,1.5$, respectively. (lower part) The dependence of the Wiedemann-Franz coefficients as defined in (16) on the magnetic field for three values of temperature. For better visibility the curves for $T=2$ have been divided by 10 and those for $T=1.5$ by 4 . The diagonal components $W^{x x}$ are plotted by the thick lines and the off-diagonal $W^{x y}$ by thin lines. We set $r_{h}=T$.

In principle it is not clear how to define the Wiedemann-Franz tensor. The definition we accepted previously [11] relied on the simple division of the respected components of transport coefficients i.e. $\tilde{W}^{i j}=$ $\kappa^{i j} /\left(\sigma^{i j} T\right)$. This definition is acceptable on phenomenological grounds but theoretically it is not satisfactory. More formal definition of the Wiedemann-Franz matrix $W$ should make use of tensorial character of both charge and thermal conductivities. Thus we propose to define the Wiedemann-Franz tensor as a tensor product

$$
W^{i j}=\kappa_{l}^{i}\left(\sigma^{-1}\right)^{l j} / T \text {. }
$$

Importantly this definition of $W$ preserves the symmetry observed for other transport coefficients with the diagonal element of the $W$ tensor being symmetric and offdiagonal one anti-symmetric function of both, the magnetic field and charge density. For vanishing $n$ or $B$ the off-diagonal component of $W$ vanishes, as $\kappa^{x y}$ vanishes in this limit. 


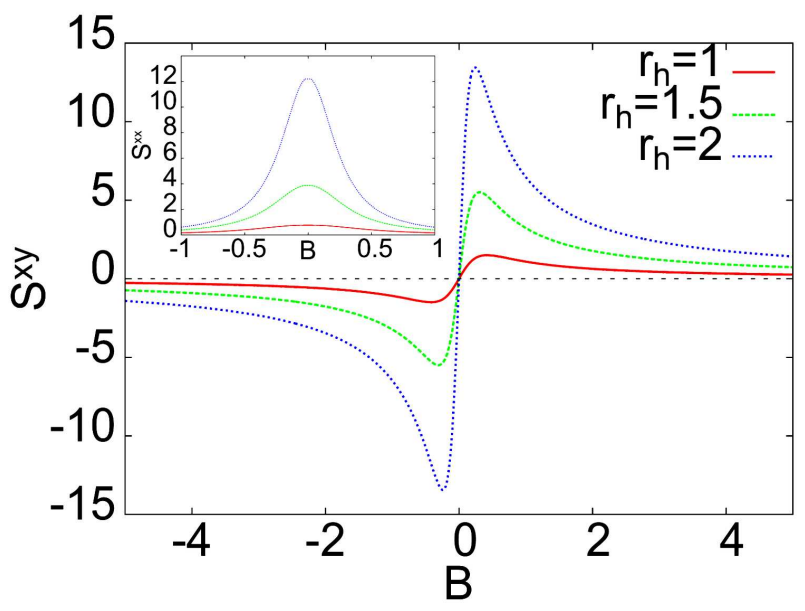

Fig. 3. Magnetic field dependence of the Nernst coefficient $S^{x y}$ for three values of the $r_{h}$ parameter related to the temperature $T$ by $T=r_{h} /(2 \pi)$. The inset shows similar dependence of the Seebeck transport coefficient $S^{x x}$.

We define thermoelectric tensor $S^{i j}$ in analogy to the definition accepted for narrow band semiconductors. First we set $E_{B}=E_{F}$, define total conductivity tensor $\sigma^{i j}=\sum_{a, b}^{F, B} \sigma_{a b}^{i j}$ and find $S^{i j}=\left(\sigma^{-1}\right)_{l}^{i} \alpha^{l j}$. Both, the Seebeck $S^{x x}$ and the Nernst $S^{x y}$ coefficients are plotted as functions of magnetic field in Fig. 3.

\section{Conclusion}

Assuming that close to the particle-hole symmetry point the electrons in three-dimensional materials with the Dirac spectrum are strongly interacting particles, we have used holographic analogy to calculate magnetic field dependent transport coefficients under the electric field and temperature bias. The obtained results show marked similarities to those measured for $\mathrm{Cd}_{3} \mathrm{As}_{2}$ compounds [17-19] and other materials $[13,14]$. The quantitative agreement between our results and the experimental data could probably be achieved if one considered the Landau quantization of the spectrum in strong fields, an effect not taken into account in the present approach. On the experimental side, the Landau quantization leads to the quantum oscillations clearly visible in experimental data [13, 14, 17-19] at high fields. As possible experimental test of the holographic approach to condensed matter systems we propose to measure two components of the magnetic field dependent Wiedemann-Franz ratios defined in Eq. (16) and compare their symmetries with those visible in Fig. $2 \mathrm{~b}$.

\section{Acknowledgments}

M.R. was partially supported by the grant no. DEC2014/15/B/ST2/00089 of the National Science Center and KIW by the grant DEC-2014/13/B/ST3/04451.

\section{References}

[1] J.M. Maldacena, Adv. Theor. Math. Phys. 2, 231 (1998).

[2] A.G. Green, Contemp. Phys. 54, 33 (2013).

[3] S.S. Gubser, I.R. Klebanov, A.M. Polyakov, Phys. Lett. B 428, 105 (1998).

[4] A. Donos, J.P. Gauntlett, J. High Energy Phys. 4, 40 (2014).

[5] A. Donos, J.P. Gauntlett, J. High Energy Phys. 6, 7 (2014).

[6] A. Donos, J.P. Gauntlett, J. High Energy Phys. 11, 081 (2014).

[7] A. Amoretti, A. Braggio, N. Maggiore, N. Magnoli, D. Musso, J. High Energy Phys. 09, 160 (2014).

[8] Y. Seo, G. Song, P. Kim, S. Sachdev, S.-J. Sin, Phys. Rev. Lett. 118, 036601 (2017).

[9] S. Sachdev, Ann. Rev. Condens. Matter Phys. 3, 9 (2012).

[10] J. Crossno, J.K. Shi, K. Wang, X. Liu, A. Harzheim, A. Lucas, S. Sachdev, P. Kim, T. Taniguchi, K. Watanabe, T.A. Ohki, K.C. Fong, Science 351, 1058 (2016)

[11] M. Rogatko, K.I. Wysokiński, J. High Energy Phys. 1, 78 (2018).

[12] M.S. Foster, I.L. Aleiner, Phys. Rev. B 79, 085415 (2009).

[13] M. Matusiak, J.R. Cooper, D. Kaczorowski, Nature Commun. 8, 15219 (2017).

[14] M. Neupane, I. Belopolski, M.M. Hosen, D.S. Sanchez, R. Sankar, M. Szlawska, S.-Y. Xu, K. Dimitri, N. Dhakal, P. Maldonado, P.M. Oppeneer, D. Kaczorowski, F. Chou, M.Z. Hasan, T. Durakiewicz, Phys. Rev B 93, 201104 (2016).

[15] T. Cichorek, private communication.

[16] J. Gooth, A.C. Niemann, T. Meng, A.G. Grushin, K. Landsteiner, B. Gotsmann, F. Menges, M. Schmidt, C. Shekhar, V. Süss, R. Hühne, B. Rellinghaus, C. Felser, B. Yan, K. Nielsch, Nature 547, 324 (2017).

[17] T. Liang, Q. Gibson, M.N. Ali, M. Liu, R.J. Cava, N.P. Ong, Nature Mater. 14, 280 (2015).

[18] T. Liang, J. Lin, Q. Gibson, T. Gao, M. Hirschberger, M. Liu, R.J. Cava, N.P. Ong, Phys. Rev. Lett. 118, 136601 (2017).

[19] P. Cheng, C. Zhang, Y. Liu, X. Yuan, F. Song, Q. Sun, P. Zhou, D.W. Zhang, F. Xiu, New J. Phys. 18 , 083003 (2016). 